\title{
Genotype by Environment Interaction of Soybean (Glycine Max L. Merrill) For Yield and Yield Components in the Savanna
}

\author{
Ochigbo, A.E. ${ }^{l}$, L.L. Bello ${ }^{l}$ and T. Vange. ${ }^{l}$ \\ ${ }^{I}$ (Department of Plant Breeding and Seed Science, University of Agriculture, Makurdi. Nigeria.)
}

\begin{abstract}
Genotype-by-environment interaction was exploited using ten genotypes in four locations covering Northern and Southern Guinea Savanna ecological zone in Nigeria. In each of the locations the experiment was laid out in a randomized complete block design (RCBD) with three replications for two years 2013 and 2014 during the rainy season.GGE (genotype main effect $(G)$ and genotype by environment $(G E)$ interaction) bi-plot graphical tool was used to analyze the multi-environment trials (MET) data. The partitioning of the GE interaction showed that PC1 and PC2 accounted for 59.2\% and 26.2\% of the GGE sum of squares respectively. This also explained a total of $85.4 \%$ of the GEI variation. Two mega-environments were characterized with genotypes TGX 1904-6F, TGX 1935-3F, TGX 1440-1E and TGX 1984-22F falling into them. TGX 1448-2E and TGX 1895-35F appeared to be overall best genotypes combining high stability and yield though they did not fall into mega-environment could be recommended for use in breeding programmes.
\end{abstract}

Keywords: AMMI, GGE bi-plot, Glycine max, MET, principal component

\section{Introduction}

Genotype $\mathrm{x}$ environment (GxE) interaction is of notable importance in the development and evaluation of new varieties. Phenotype is a mixture of genotype (G) and environment (E) components and also an interaction between genotype by environment. Consequently, multi-environment trials (MET) are conducted by plant breeders to evaluate yield stability performance of genetic materials under varying environmental conditions [1]. A genotype grown in different environments will most times show fluctuations in yield performance. These changes or differences are influenced by the different environmental conditions and are referred to as genotype by environment. G x E interaction should be exploited either by selecting superior genotype for each specific target environment or it could be avoided by selecting widely adapted and stable genotype across wide range of environments.

Numerous methods have been developed to reveal the pattern of GxE like regression [2], coefficient of variability [3] and additive main effect and multiplicative interaction (AMMI) [4]. All these methods are commonly used to analyze MET data. Yan [1] proposed another method known as GGE bi-plot for graphical display of GE interaction pattern of MET data with many advantages as uses functions from many of the other methods jointly. In general total phenotypic variation, E explains most of the variation and $\mathrm{G}$ and GE are usually small [5]. However, only the $\mathrm{G}$ and GxE interaction are relevant to cultivar evaluation especially when GxE interaction is identified as repeatable. GGE-biplot allows for visual examination of relationships among test environments, genotypes and genotype by environment interactions. It is an effective tool for -

- Identifying mega-environment (which-won-wherepattern) whereby specific genotypes can be recommended to specific environments [6]

- Evaluation of genotypes for mean performance and stability and

- Evaluation of environment (discriminating among genotypes in target environments) [7].

The objectives of this study are to evaluate soybean varieties under different growing environment with a view to determine the effect of GxE interaction on yield of some released varieties and identify stable varieties for specific environments.

\section{Materials And Methods}

\subsection{Study area, design and management}

Ten soybean varieties belonging to different maturity groups were used for this study presented in TABLE 1. These varieties were selected from those released to the University of Agricture, Makurdi from IITA and NCRI. The study areas were four sites viz Makurdi, Yandev and Otobi situated in the Southern Guinea Savanna and Jos situated in the Northern Guinea Savanna ecological zone of Nigeria. This was a large area of the Nigerian Savanna zone covering Latitude $7^{\circ} 22^{\prime} 19.99^{\prime \prime}-9^{\circ} 57^{\prime} 7.33^{\prime \prime} \mathrm{N}$ and Longitude $8^{\circ} 3{ }^{\prime} 34.21$ 'E. These locations are representative of soybean production area of Nigeria with slight difference in their soil type and climatic conditions. The experiment was laid out in a randomized complete block design (RCBD) with three replications in each of the four locations used for this trial. The field was cleared, ploughed, harrowed and ridges 
made. Each plot measured $3 \mathrm{~m}$ x $2 \mathrm{~m}$ consisting of four ridges. Soybean seeds of good quality were planted using drill method such that there was $50-75 \mathrm{~cm}$ between rows and $5 \mathrm{~cm}$ within rows. With this spacing, each plot had a plant population of a 400,000 plants per hectare. In 2013, planting was done between $22^{\text {nd }}$ and $25^{\text {th }}$ July while in 2014, planting was done between $10^{\text {th }}$ and $15^{\text {th }}$ July starting with Makurdi then Yandev, Otobi and Jos. Soybean produces over a wide range of planting dates if moisture is available. The trials were routinely managed in terms of weeding and other agronomic practices till the seeds were collected.

The middle rows in each plot were used for data collection for quantitative traits. The plot yield data were converted into kilogrammes per hectare. The data collected was subjected to analysis of variance. Least significant difference (LSD) was used to separate the means where significance existed at $5 \%$ level of probability $(\mathrm{P}<0.05)$. This test discriminates the genotypes identify superior ones based on the traits of interest but it does not indicate which genotype possess more contribution to the genotype $\mathrm{x}$ environment interaction. Stability analysis was then carried out using the AMMI (additive main effect and multiplicative interaction) model as described by [14 ] and GGE-bi-plot analysis was also done. AMMI model integrates analysis of variance (ANOVA) and principal component analysis (PCA) into a unified approach.

Table 1:- Description of Genotypes Used for the Study

\begin{tabular}{|l|l|l|}
\hline Genotype & Maturity Group & Days to maturity \\
\hline TGX 1440 - IE & Late & 120 \\
\hline TGX 1448-2E & Medium & $100-110$ \\
\hline TGX 1485- ID & Early & 90 \\
\hline TGX 1835-10E & Early & 90 \\
\hline TGX 1866-7F & Late & 120 \\
\hline TGX 1895-35F & Late & 120 \\
\hline TGX 1904-6F & Medium & $100-110$ \\
\hline TGX 1935-3F & Medium & $100-110$ \\
\hline TGX 1984-5F & Medium & $100-110$ \\
\hline TGX 1984-22F & Medium & $100-110$ \\
\hline
\end{tabular}

\section{Results And Discussion}

Mean squares estimates for yield and other yield attributes of soybean varieties used for the study in four locations for two years is presented in TABLE 2. There was no significance observed for the interaction between genotype, year and location for all the traits studied. Year x genotype also showed no significance between years across all locations studied. Location $\mathrm{x}$ year was also not significant for all the traits studied but Rep (Loc x year) showed significance for all the traits except for plant height at 4WAP and hundred seed weight. Genotype $(\mathrm{G})$ showed significance at $5 \%$ level or probability among themselves for all the traits except for pods/plant and seeds/plant. From the table also the year (Y) showed significance for all traits except for pods/plant, seeds/plant and yield. Only the location (L) mean square was significant for all the traits at $5 \%$ level of probability. The coefficient of variability for all the traits studied ranged between $6.881 \%$ for hundred seed weight and $36.163 \%$ for pods per plant.

In this study, the additive main effect and multiplicative interaction (AMMI) analysis of variance shows that the environmental variance was significant and higher than both the genotype and genotype by environment interaction (GEI) variance. This result also showed that the environment (E) main effect was the most important source of variance due to its large contribution to the total sum of squares and mean squares for all the traits evaluated. Variation due to genotype was larger than that due to GEI indicating that differences among genotypes vary across locations. That is to say, genotypes reacted differently from one environment to another as the environmental conditions were not the same coupled with other factors from one location to another. Similarities between locations and differences within locations in rainfall patterns also could have been responsible for performance of genotypes and this suggests that climatic information might be useful in the clarification of genotype trial interaction. Similar observations were obtained by [9] and [10].

The AMMI model combines additive components in a single model for the main effects of the genotypes and environments, as well as multiplicative components for the interaction effect. For any particular genotype by environment, the main effect equals the genotype mean plus the environment mean minus the grand mean. The interaction is the genotype principal component axis (PCA) score multiplied by the environment score. From this study the first principal component (PC1) was very high and explained $59.2 \%$ of the interaction while the second principal component (PC2) explained $26.2 \%$ of the interaction. Both principal components captured $85.4 \%$ of the genotype by environment interaction and represent also $85.4 \%$ of the total sum of square of the interaction so it is possible to explain that much of the GXE interaction. This implies that the interaction of the ten genotypes studied in the four environments was predicted by the first two principal components of the genotypes and environments as stated also by Gauch \& Zobel [11],[12]. This also suggests that the soil and climatic conditions of the various environments, interfered with the performance of the genotypes especially as 
the (PCA) axes of the AMMI Model usually relates to length of growing environment, temperature changes, variation in soil or a combination of all factors [13].

The GGE bi-plot analysis of the ten soybean varieties evaluated in four environments with respect to yield is presented in Fig.1. The first two principal components (PC) explained $85.4 \%$ of the total variation. This figure explicitly displays the polygon view of the GGE- bi-plot with "which-won-where" pattern to identify the best genotypes for each environment. The polygon is formed by connecting the markers of the genotypes that are furthest away from the bi-plot origin such that the other genotypes fall within the polygon. The rays are lines that are perpendicular to the sides of the polygon or their extension [5]. The polygon was formed by connecting $\mathrm{Tg} 1, \mathrm{Tg} 7, \mathrm{Tg} 8, \mathrm{Tg} 5$ and $\mathrm{Tg} 9$ causing them to be at the vertex and all other genotype contained within the polygon. The rays formed are numbered 1 to 5 . Rays 1 and 2 are perpendicular to the side that connects genotype $\mathrm{Tg} 5$ and $\mathrm{Tg} 8$ while ray 3 is perpendicular to the side that connects genotype $\mathrm{Tg} 5 \mathrm{and} \mathrm{Tg} 9$ and so on to ray 5 being perpendicular to the side that connects genotypes $\operatorname{Tg} 1$ and $\mathrm{Tg} 7$. These perpendicular lines divide the bi-plot into several sectors. There are five sectors in Fig. 1 and the four environments in this study fall into two of the sectors. The environment group within each sector and the variety at the polygon's extremity characterized the mega-environment [5]. Thus, two mega-environments were characterized, one with Makurdi and Otobi, while the other has Jos and Yandev.

AMMI and GGE bi-plot models are defined powerful tools for effective analysis and interpretation of multi-environment data structure in breeding programs [1]. Gauch Junior[14] reviewed AMMI \& GGE bi-plot analysis and concluded that AMMI mega-environment graph incorporated more of the genotype main effect and captured more of the genotype by environment interaction. The GGE bi-plot graph thereby displays the 'whichwon-where' (i.e. the best genotype in each environment) pattern more accurately for complex data sets. It is a summary of the GEI pattern of a multi-environment yield trial data. In this study, the 'which-won-where' was displayed in the graph showing sites and genotypes in Fig. 1. Five rays divided the biplot into five sections and the four sites fell into two of these sections. The vertex genotype for each quadrant is the ones that gave the highest yield for the environments they fell within them. From this figure, the best performers for Makurdi and Otobi are TGX 1904-6F and TGX 1935-3F while TGX 1440-1E and TGX 1984-22F being at the vertices performed best in Jos and Yandev. Though there were five sectors in all, two mega-environments were identified. The remaining three sectors had no environment within them and contain the following genotypes TGX 1485-1D, TGX 1835-10E and TGX 1866-1F. Only TGX 1866-1F was on the vertex. Genotypes in vertex without environment performed poorly in all the sites [15]. However, genotypes within the polygon, particularly those located near the biplot origin were less responsive than the genotypes on the vertices and the ideal genotype would be one closest to the origin ([12]; [15] and an ideal test environment should be both discriminating and representative [16]. An ideal environment probably does not exist in reality but if the genotype does well or better in a particular environment, it would be used as a reference point. Fig. 2 presents the GGE bi-plot for soybean yield grown in four locations showing similarities among test environments in discriminating the genotypes. By plotting both genotypes and environments on the same graph, the association between the genotypes and the environments became more obvious. The lines that connect the bi-plot origin and the markers for the environments are called environment vectors. The angle between the vectors of two environments is related to the correlation coefficient between them. The cosine of the angle between the vectors of the two environments approximates the correlation coefficient between them [5]. The small circle near Otobi environment indicates average environment which is defined by the intercept of PC1 and PC2 scores of the environment [17]. In this figure, the line that passes through the bi-plot origin and the average environment with single arrow is called the average environment coordinate (AEC). This line is also the ordinate. Displacement along the ordinate exhibites a difference in the principal components. The additive part of the AMMI equals the $\mathrm{G}$ mean plus the E mean minus the grand mean and the multiplicative part i.e interaction effect, is the product of $\mathrm{G}$ and $\mathrm{E}$. The genotypes $\operatorname{Tg} 6, \operatorname{Tg} 7, \operatorname{Tg} 8$ and $\operatorname{Tg} 10$ were generally high yielding since AMMI placed them on the right hand side of the midpoint of the axis. In relation to the ideal genotype which is TGX 1448-2E as indicated by the bi-plot, genotypes TGX 1485-1D, TGX 1835-10E and TGX 1895-35F were closest to the ideal genotype position. This suggests that these genotypes were more stable than all other genotypes in all the study environments. Their yield could also be comparable to those in the vertices in the other environments. An ideal genotype is one that combines yield with stability performance [12]; [13].

\section{Conclusion}

This result revealed that TGX 1448-2E, TGX 1485-1D, TGX 1835-10E and TGX 1895-35F were most stable genotypes because their interaction with the environment was not enough to hinder yield as indicated by the IPCA scores of zero or near zero, suggesting that these genotypes could be cultivated in any of the four environments for their stability. TGX 1448-2E and TGX 1895-35F appeared to be the overall best genotype combining high stability with yield therefore it could be recommended for cultivation. Genotypes with large 
Genotype By Environment Interaction Of Soybean (Glycine Max L. Merrill) For Yield And Yield...

interaction with the environment are unpredictable in performance and can only be grown in limited or specific environments.

Table 2:- Mean Square Estimates from the Analysis of Variance for Plant Height and Other Yield Components for Ten Soybean Genotypes in the Four Environments.

\begin{tabular}{|c|c|c|c|c|c|c|c|c|c|c|}
\hline$(\mathrm{SOV})$ & \multirow[b]{2}{*}{ DF } & \multicolumn{2}{|c|}{ PLANT HEIGHT } & \multicolumn{2}{|c|}{ LEAF NUMBER } & \multirow[t]{2}{*}{ BRANCHES } & \multirow[t]{2}{*}{ PODS/PLT } & \multirow[t]{2}{*}{ SEEDS/PLT } & \multirow[t]{2}{*}{ YIELD } & \multirow{2}{*}{$\begin{array}{l}100 \\
\text { SEED } \\
\text { WEIGHT }\end{array}$} \\
\hline $\begin{array}{l}\text { Source of } \\
\text { variance }\end{array}$ & & $4 \mathrm{WAP}$ & 8 WAP & 4 WAP & 8 WAP & & & & & \\
\hline Location (L) & 3 & $1095.47^{*}$ & $1521.89^{*}$ & $423.710^{*}$ & $35537.45^{*}$ & $70.085^{*}$ & $43736.298^{*}$ & $21071.108^{*}$ & $136291294.2^{*}$ & $17.179^{*}$ \\
\hline Year $(\mathrm{Y})$ & 1 & $301.504^{*}$ & $1399.98^{*}$ & $552.521^{*}$ & $2167.08^{*}$ & $214.742^{*}$ & $5306.019^{*}$ & 1035.717 & 2427277.1 & $20.945^{*}$ \\
\hline $\mathrm{Y} \times \mathrm{L}$ & 3 & 1.318 & $0.57^{*}$ & 0.519 & 0.848 & 0.282 & 897.437 & 3.721 & 1570.9 & 2.229 \\
\hline $\operatorname{Rep}(Y x L)$ & 16 & 5.979 & $129.55^{*}$ & 2.396 & $1033.99^{*}$ & $2.759^{*}$ & $2951.141^{*}$ & $1202.610^{*}$ & $4975817.7^{*}$ & 1.337 \\
\hline $\begin{array}{l}\text { Genotype } \\
\text { (G) }\end{array}$ & 9 & $42.463^{*}$ & $828.74^{*}$ & $48.668^{*}$ & $1750.14^{*}$ & $6.457^{*}$ & 1490.295 & 446.948 & $2996308.4^{*}$ & $4.151^{*}$ \\
\hline$Y \times G$ & 9 & 0.101 & 0.186 & 0.153 & 0.84 & 0.065 & 5.625 & 1.986 & 696.2 & 0.035 \\
\hline $\mathrm{L} \times \mathrm{G}$ & 27 & $17.740^{*}$ & 109.76 & $20.184^{*}$ & $465.57^{*}$ & 1.530 & $1698.00^{*}$ & 710.420 & $2090665.6^{*}$ & $3.977^{*}$ \\
\hline YxLxG & 27 & 0.171 & 0.259 & 0.127 & 0.344 & 0.075 & 10.45 & 2.041 & 297.1 & 0.039 \\
\hline Error & 144 & 3.466 & 18.052 & 2.697 & 125.621 & 0.629 & 503.842 & 136.671 & 418510.0 & 0.608 \\
\hline $\mathrm{CV} \%$ & & 10.329 & 10.038 & 9.911 & 14.697 & 7.54 & 36.163 & 31.698 & 23.306 & 6.881 \\
\hline $\mathrm{R}^{2}$ & & 0.901 & 0.877 & 0.880 & 0.895 & 0.863 & 0.772 & 0.844 & 0.905 & 0.731 \\
\hline
\end{tabular}

ns $\quad=$

significance at $\mathrm{P} \leq 0.05$

not significant

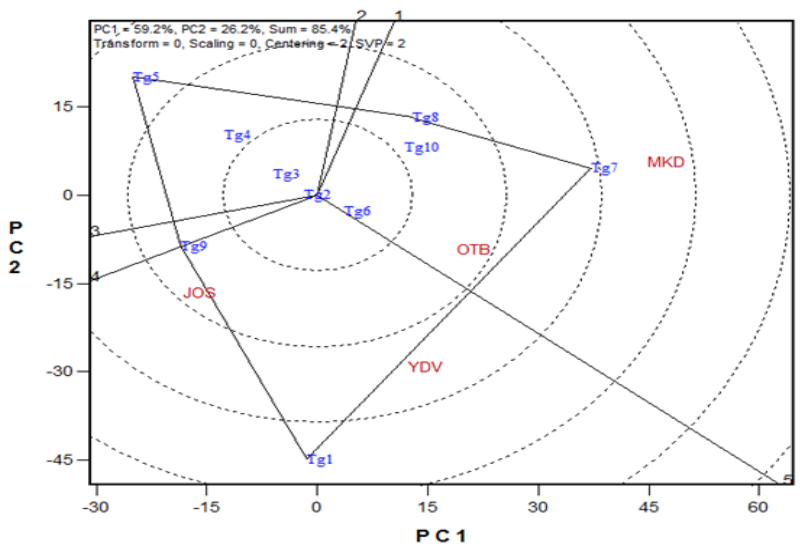

Figure 1: POLYGON VIEW OF GGE BIPLOT (Which won where)

Tg1-TGX1440-1E Tg2-TGX1448-2E Tg3-TGX1485-1D Tg4-TGX1830-10E Tg5-TGX1866-1F Tg6-TGX1895-35F Tg7-TGX1904-6F Tg8-TGX1935-3F Tg9-TGX1984-22F Tg10-TGX1984-5F

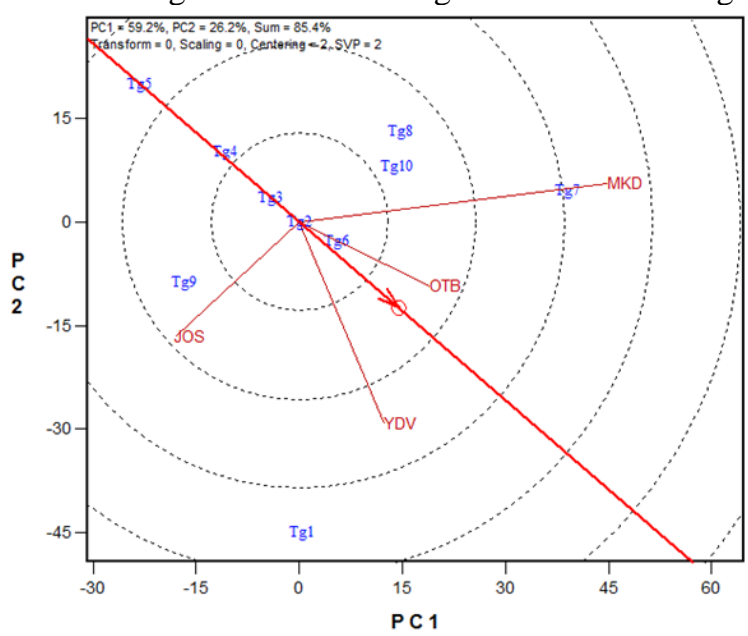

Figure 2: THE ENVIRONMENT VECTOR VIEW OF THE GGE BI-PLOT SHOWING SIMILARITIES AMONG TEST LOCATIONS IN DISCRIMINATING THE GENOTYPES

Tg1-TGX1440-1E Tg2-TGX1448-2E Tg3-TGX1485-1D Tg4-TGX1830-10E Tg5-TGX1866-1F Tg6-TGX1895-35F Tg7-TGX1904-6F Tg8-TGX1935-3F Tg9-TGX1984-22F Tg10-TGX1984-5F 


\section{References}

[1]. Yan, W. Hunt, L. A. Sheng, Q; and Z. Szlavnics. (2000). Cultivar Evaluation and Mega-Environment Investigation Based on the GGE Bi-plot. Crop Sci. 40:597-605.

[2]. Eberhart, S. A. and Russell, W. A. (1966). Stability Parameters for Company Varieties. Crop science 6:36-40.

[3]. Francis, T. R. and Kannenberg, L. W. (1978). Yield Stability Studies in Short-Season Maize. A Descriptive Method of Grouping Genotypes. Canadian Journal of Plant Science 58:1029-1034.

[4]. Gauch, H. G. (1992). Statistical Analysis of Regional Yield Trials: AMMI Analysis of Factorial Designs. Elsevier, Amsterdam. Netherlands. Pp. 220-230.

[5]. Yan, W. (2002). Singular Value Partitioning in Bi-plot Analysis of Multi-Environment Trial Data. Agronomy Journal 94:990-996.

[6]. Yan, W. and Tinker, N. A (2006). Bi-plot Analysis of Multi-Environment Trial Data. Principles and Applications. Canadian Journal of Plant Sci. 86:623-645.

[7]. Ding Meimei, Bruce Tier and Weikai Yan (2007). Application of GGE Bi-plot Analysis to Evaluate Genotype (G), Environment (E) and G x E Interaction on P. radiation:- A Case Study. Paper presented at Australian Forest Genetics Conference. Hobart Australia. 11-14 April 2007. Pg1-15.

[8]. Gauch, H. G. Piepho, H. P. and P. Annicchiarico (2008). Statistical Analysis of Yield Trials by AMMI and GGE: Further Considerations. Crop sci. 48:866-889.

[9]. Ceyhan, E. Kahraman, A. Ates, M. K. and Karadas, S. (2012). Stability Analysis on Seed Yield and Its Components in Peas. Bulgarian Journal of Agricultural sciences 18:905-911.

[10]. Kaya, Y., Akcura, M. and Taner, S. (2006). GGE Bi-plot Analysis of Multi- Environment Yield Trials In Bread Wheat. Turkish Journal of Agriculture 30:325-337.

[11]. Gauch, H. G. and Zobel, R. W. (1996). AMMI Analysis of Yield Trials: In M. S. Kang and H. G. Gauch, Eds. Genotype-byEnvironment Interaction, CRC Press, New York. Pg 416.

[12]. Nwangburuka, C. C., O. B. Kehinde, D. K. Ojo and D. A. Denton (2011). Genotype x Environment Interaction and Seed Yield Stability in Cultivated Okra using Additive Main effect and Multiplicative Interaction (AMMI) and Genotype and Genotype $\mathrm{x}$ Environment (GGE) Interaction. Applied Science Research 3(4)193-205.

[13]. Ado, S. G. and Ishiyaku, M. F. (1999). Genotype x Environment Interaction In Pepper (Capsicum annuum L.) Evaluation Trials In the Nigerian Savanna. Nigerian J. Genetics 14:78-86.

[14]. Ishaq, M. N., H. Agrama and A. Adeleke (2015). Exploring Genotype x Environment Interaction In Soybean Breeding In Nigeria. Int. Journal of Adv. Research in Biological sciences 2(1): 24-32.

[15]. Alake, C. O. and O. J. Ariyo (2012). Comparative Analysis of Genotype x Environment Techniques in West African Okra (Abelmoschus caillei A. Chav Stevels). Journal of Agriculture Science 4(4)135-150.

[16]. Yan, W. and Kang, M. S. (2003). GGE Bi-plot Analysis - A Graphical Tool for Breeders, Geneticists and Agronomists. First edition CRC Press, Florida. Pp. 271. 